\title{
BMJ Open Use of wind-up fetal Doppler versus Pinard for fetal heart rate intermittent monitoring in labour: a randomised clinical trial
}

\author{
R Byaruhanga, ${ }^{1} \mathrm{D}$ G Bassani, ${ }^{2}$ A Jagau, ${ }^{3} \mathrm{P}$ Muwanguzi, ${ }^{4} \mathrm{~A}$ L Montgomery, ${ }^{2}$ \\ J E Lawn ${ }^{5}$
}

To cite: Byaruhanga $R$, Bassani DG, Jagau A, et al. Use of wind-up fetal Doppler versus Pinard for fetal heart rate intermittent monitoring in labour: a randomised clinical trial. BMJ Open 2015;5:e006867. doi:10.1136/bmjopen-2014006867

- Prepublication history for this paper is available online. To view these files please visit the journal online (http://dx.doi.org/10.1136/ bmjopen-2014-006867).

RB and DGB are joint first authors.

Received 14 October 2014 Revised 23 December 2014 Accepted 29 December 2014

CrossMark

For numbered affiliations see end of article.

Correspondence to Dr AL Montgomery; ann.montgomery@sickkids.ca

\section{ABSTRACT}

Objectives: In resource-poor settings, the standard of care to inform labour management is the partograph plus Pinard stethoscope for intermittent fetal heart rate (FHR) monitoring. We compared FHR monitoring in labour using a novel, robust wind-up handheld Doppler with the Pinard as a primary screening tool for abnormal FHR on perinatal outcomes.

Design: Prospective equally randomised clinical trial. Setting: The labour and delivery unit of a teaching hospital in Kampala, Uganda.

Participants: Of the 2042 eligible antenatal women, 1971 women in active term labour, following uncomplicated pregnancies, were randomised to either the standard of care or not.

Intervention: Intermittent FHR monitoring using Doppler.

Primary outcome measures: Incidence of FHR abnormality detection, intrapartum stillbirth and neonatal mortality prior to discharge.

Results: Age, parity, gestational age, mode of delivery and newborn weight were similar between study groups. In the Doppler group, there was a significantly higher rate of FHR abnormalities detected (incidence rate ratio (IRR) $=1.61,95 \% \mathrm{Cl} 1.13$ to 2.30 ). However, in this group, there were also higher though not statistically significant rates of intrapartum stillbirths (IRR=3.94, 0.44 to 35.24 ) and neonatal deaths (IRR=1.38, 0.44 to 4.34 ).

Conclusions: Routine monitoring with a handheld Doppler increased the identification of FHR abnormalities in labour; however, our trial did not find evidence that this leads to a decrease in the incidence of intrapartum stillbirth or neonatal death.

Trial registration number: Clinical Trails.gov (1000031587).

\section{INTRODUCTION}

Approximately, $44 \%$ of all child deaths under the age of 5 years occur in neonates ( $<28$ days of age). ${ }^{1}$ The third largest cause of neonatal mortality is intrapartum-related hypoxia (formerly called 'birth asphyxia')

\section{Strengths and limitations of this study}

- This is the first study to use current monitoring guidelines to evaluate Doppler versus Pinard in improving stillbirth and neonatal outcomes.

- A major strength of this study includes the prospective and randomisation design.

- We were unable to perform secondary screening of suspected fetal hypoxia through the use of cardiotocography and we could not confirm the presence of fetal hypoxia acidaemia via fetal blood scalp sampling and cord blood gases; therefore, we were unable to assess if the identification (or lack of identification) of abnormal fetal heart rate was correlated with the presence of fetal hypoxia acidaemia.

- We were unable to exclude some cases where the underlying cause of death was other than fetal hypoxia (eg, congenital anomalies, early onset sepsis) due to diagnostic limitations in differentially diagnosing these cases.

resulting in an estimated 660000 neonatal deaths per year globally ${ }^{1}$ and an additional 414000 children who survive with disability. ${ }^{2}$ There are also an estimated 1.02 million intrapartum stillbirths, almost all in lowincome and middle-income countries. ${ }^{3}$ This burden is highest in areas of the world where the probability of quality of care at birth is the lowest. ${ }^{4}$ In order to reduce the incidence of intrapartum-related stillbirths and neonatal deaths, it is necessary to assess fetal well-being in labour with routine monitoring of the fetal heart rate (FHR), linked to rapid and effective management with resuscitative measures or prompt delivery, and provision of neonatal resuscitation if needed.

Characteristic FHR changes often precede brain injury via a process of progressive fetal hypoxic acidaemia. ${ }^{5}$ Intermittent auscultation as a primary screening tool to monitor fetal well-being is the recommended 
standard of care for women experiencing uncomplicated deliveries. ${ }^{6-9}$ One method of intermittent auscultation uses the Pinard Fetal Stethoscope (Pinard), a trumpet shaped horn, to monitor the FHR, and is widely adopted as the standard of care in resource-poor settings since it is low cost and does not require a power source or repairs. The difficulties posed in using a Pinard are generally not conducive to a busy labour ward. It requires additional time to precisely locate the fetal heart as the heart is only audible within a very narrow area of the woman's abdomen; it requires that the surrounding area be quiet in order to hear the fetal heart. The reading can be unreliable in obese women, and it requires the midwife to place her ear in close proximity to the woman's pubic area. In addition, the midwife usually counts the FHR for a short time, for example, $15 \mathrm{~s}$, and multiplies to reach bpm, further decreasing accuracy and introducing calculation errors. The handheld Doppler ultrasound FHR monitor (Doppler) detects FHR and provides a steady state number per minute, as well as audible auscultation of the FHR. It requires a reliable power source and may need repairs, and is more costly than a Pinard. However, it permits the midwife to quickly locate the FHR, allows others, including the mother-to-be, to hear the FHR and permits her to remain in any comfortable position while being assessed, permits the midwife to assess the FHR as well as communicate to the woman the status of her baby, and has been shown to be preferred by women over the use of the Pinard. ${ }^{8}{ }^{10}$ A rugged, wind-up, handheld Doppler FHR monitor (Doppler), developed by Power-free Education Technology (Pet.og.za) showed, in initial field tests, to be accurate and acceptable to mothers as well as midwives in low-resource settings. ${ }^{11}{ }^{12}$ It uses a hand crank to generate 2:30 min of use for every $30 \mathrm{~s}$ of cranking.

While there have been several studies showing reduced intervention and no improved outcomes in the use of the intermittent (Pinard or Doppler) versus continuous cardiotocography (CTG) monitoring as the primary screening tool in uncomplicated deliveries, ${ }^{6}{ }^{13}$ there is little research on outcomes in intermittent monitoring comparing Doppler versus Pinard. A single study by Mahomed et $a l^{14}$ using a monitoring protocol of $10 \mathrm{~min}$ every half-hour found higher detection of FHR abnormalities and better perinatal outcomes in the intermittent auscultation Doppler group compared with the Pinard group.

We aimed to use a randomised trial design to compare the primary screening methods of FHR monitoring (Doppler as intervention vs Pinard as standard of care) on incidence of detection of FHR abnormalities, and on the incidence of intrapartum stillbirth and neonatal mortality in the first $24 \mathrm{~h}$ after delivery.

\section{METHODS}

Study design and participants

We undertook this randomised controlled trial at St. Raphael of St. Francis Nsambya Hospital, a periurban private not-for-profit hospital in Kampala, Uganda. It is a teaching hospital that manages 7500 deliveries annually. CTG and fetal blood gas sampling to support labour management, and epidural pain medication are not available. Oxytocin augmentation and caesarean delivery rates are $40 \%$ and $20 \%$, respectively. The standard of care for intrapartum FHR monitoring is by intermittent auscultation using the Pinard.

Women were requested to participate during an antenatal care appointment. This consent was reconfirmed in labour provided the women presented in labour with a singleton pregnancy, in a cephalic position, at term or post-term ( $>37$ weeks gestation). Women were excluded if they were already in second stage of labour on admission or presented with a condition that, according to the doctor on duty, contraindicated labouring (eg, antepartum haemorrhage); if there was a diagnosis of intrauterine fetal death on admission; or if the woman was admitted for an elective caesarean delivery. Participants were presented with information about the study and agreeing participants provided written consent. Registration of our protocol with ClinicalTrials.gov occurred before participant enrolment started, but due to an administrative error with our institution's Clinical Research Services Unit, the protocol was only released to the public after the completion of the study. Documentation from the Chair of our independent Research Ethics Board was provided to BMJ Open attesting to the version of the protocol provided to them prior to the start of enrolment.

\section{Randomisation}

Women were equally randomised to one of the two study methods using sequentially numbered, opaque sealed envelopes. Study participants and care providers were not blinded to the intervention. Data were collected from the patient's partograph and from the hospital's routine neonatal mortality audit data, when applicable.

\section{Procedures}

The standard of care for intrapartum monitoring relied on partograph and FHR monitoring with the Pinard. Our prestudy training addresses deficiencies in monitoring standards (acceptable range for FHR, recognition of accelerations, decelerations and change in baseline). We developed a training module entitled 'Helping Babies Survive Labour' modelling on the 'Helping Babies Breathe' visual materials and learning approach. The technical basis was from WHO and Canadian Obstetric Society protocols. ${ }^{5}$ All midwives and doctors were then given this in-service training for half a day. FHR monitoring was undertaken every $30 \mathrm{~min}$ in the first stage of labour; every $15 \mathrm{~min}$ in the second stage before pushing; and every $5 \mathrm{~min}$ in the second stage when pushing and for 1 min immediately after a contraction. The baseline FHR was recorded as a single number rather than a range, in the unit of bpm. The FHR rhythm (regular or irregular) and absence or presence of accelerations or 
decelerations were also documented. The maternal radial pulse was simultaneously palpated to differentiate it from the FHR.

When FHR abnormalities were identified, the standard of care would be to switch from intermittent auscultation to CTG. Since CTG is not available in Nsambya Hospital, any noted FHR abnormalities were reported by the research midwife to the doctor on duty for assessment. Management following this assessment was either closer intermittent monitoring or intrauterine resuscitation with reassessment of the FHR. Intrauterine resuscitation consisted of maternal position change, administration of oxygen by mask to the mother-to-be, initiation of intravenous infusion, discontinuation of oxytocin augmentation and consideration of prompt delivery (assisted vaginal if imminent, otherwise by caesarean).

\section{Outcomes}

The primary outcome measures of interest were detection of FHR abnormality in labour (defined below), intrapartum stillbirth and neonatal deaths in the first $24 \mathrm{~h}$ of life. FHR abnormality is defined as tachycardia, bradycardia, or atypical variable, late or prolonged decelerations. Tachycardia and bradycardia are defined as baselines of $>160$ and $<110 \mathrm{bpm}$, respectively. Some features of atypical variable decelerations are abrupt FHR decelerations, lasting $>2 \mathrm{~min}$, slow return to baseline, or in the presence of tachycardia. Late decelerations are a repetitive, gradual decrease in the FHR and return to baseline, starting after the onset of the contraction, and return to baseline after the end of the contraction. Prolonged decelerations are a decrease from baseline of $>15 \mathrm{bpm}$ lasting for 2-10 min. Secondary outcomes were Apgar score less than 7 at $5 \mathrm{~min}$, admission to special care unit for intrapartum-related complications (intrapartum hypoxia, neonatal encephalopathy (NE) or meconium aspiration syndrome), diagnosis of
$\mathrm{NE}$ and delivery by caesarean. A validated and simplified scoring method was used for grading mild, moderate and severe NE. ${ }^{15}{ }^{16}$ Indications for caesarean delivery were failure to progress (as indicated by crossing of the action line on the partograph), abnormal FHR unresponsive to uterine resuscitation and identification of malpresentation in labour (eg, conversion from vertex to brow or mentum posterior).

\section{Statistical analysis}

Estimating that the use of the Doppler would reduce intrapartum stillbirth by $30 \%$ compared with the Pinard (based on the results of Mahomed et $a l^{14}$ ), with $80 \%$ power to detect at least a $30 \%$ reduction in stillbirths with $95 \%$ confidence, we would need to enrol 840 participants in each of the two comparison groups. We added $20 \%$ to the sample size for each study arm to account for loss to follow-up and statistical adjustments and stratification, resulting in 1008 participants required for each comparison group.

Data were double entered from the partograph and, where applicable, the hospital's routine neonatal mortality audit document. An interim analysis was conducted by the data safety and monitoring board at the midpoint of the data collection period. Descriptive statistics were used to describe the characteristics of the participants and their outcomes under each study arm.

We used population-averaged generalised Poisson regression modelling with robust variance to compare methods of FHR monitoring with Doppler versus Pinard on incidence rate ratio (IRR) of detection of FHR abnormalities, intrapartum stillbirth and neonatal mortality (see Barros and Hirakata ${ }^{17}$ for details of this choice over logistic regression). We conducted a subgroup analysis and qualitative reporting on the intrapartum stillbirths and pre-discharge neonatal deaths within $24 \mathrm{~h}$ and those fetuses with detected abnormal FHR.

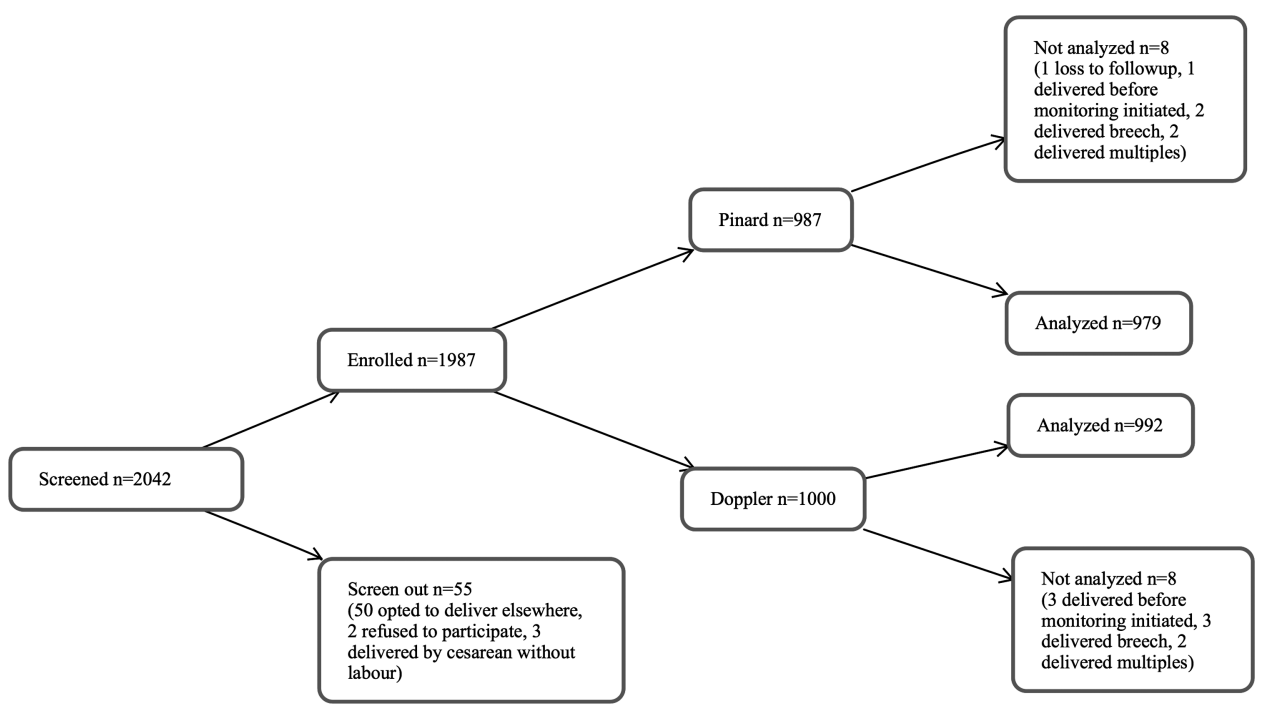

Figure 1 Trial profile. 
Table 1 Demographic, clinical and perinatal characteristics

\begin{tabular}{|c|c|c|c|}
\hline & Pinard $(n=979)$ & Doppler $(n=992)$ & p Value \\
\hline \multicolumn{4}{|c|}{ Maternal age (years) } \\
\hline Median (IQR) & $26(23-30)$ & $27(24-30)$ & 0.95 \\
\hline \multicolumn{4}{|l|}{ Marital status } \\
\hline Married & 816 (83.4) & $818(82.5)$ & \multirow[t]{2}{*}{0.60} \\
\hline Single & $163(16.7)$ & $174(17.5)$ & \\
\hline \multicolumn{4}{|c|}{ Maternal education } \\
\hline None & $9(0.9)$ & $7(0.7)$ & \multirow[t]{6}{*}{0.62} \\
\hline Primary & $93(9.5)$ & $94(9.5)$ & \\
\hline Secondary & 385 (39.3) & $423(42.6)$ & \\
\hline Vocational & $235(24.0)$ & $224(22.6)$ & \\
\hline University & 257 (26.3) & $243(24.5)$ & \\
\hline Missing & & $1(0.1)$ & \\
\hline \multicolumn{4}{|c|}{ Maternal occupation } \\
\hline Housewife & 357 (36.5) & $377(38.0)$ & \multirow[t]{5}{*}{0.80} \\
\hline Skilled worker & $84(8.6)$ & $75(7.6)$ & \\
\hline Self-employed & $271(27.7)$ & $260(26.2)$ & \\
\hline Professional & $252(25.7)$ & $262(26.4)$ & \\
\hline Other & $15(1.5)$ & $18(1.8)$ & \\
\hline \multicolumn{4}{|c|}{ Number of ANC visits } \\
\hline Median (IQR) & $4(3-5)$ & $4(3-5)$ & 0.58 \\
\hline \multicolumn{4}{|c|}{ Complication noted in pregnancy } \\
\hline Yes & $17(98.3)$ & $24(2.4)$ & \multirow[t]{2}{*}{0.29} \\
\hline No & $962(1.7)$ & $968(97.6)$ & \\
\hline \multicolumn{4}{|l|}{ Gravity } \\
\hline 1 & $334(34.1)$ & $332(33.5)$ & \multirow[t]{3}{*}{0.64} \\
\hline 2 & $422(43.1)$ & 416 (41.9) & \\
\hline$\geq 3$ & $223(22.8)$ & $244(24.6)$ & \\
\hline \multicolumn{4}{|l|}{ Parity } \\
\hline 0 & $413(42.2)$ & 395 (39.8) & \multirow[t]{3}{*}{0.31} \\
\hline 1 & $238(24.3)$ & $232(23.4)$ & \\
\hline$\geq 2$ & $328(33.5)$ & $365(36.8)$ & \\
\hline \multicolumn{4}{|c|}{ Previous perinatal death } \\
\hline Yes & $24(2.5)$ & $29(2.9)$ & \multirow[t]{2}{*}{0.52} \\
\hline No & $955(97.6)$ & $963(97.1)$ & \\
\hline \multicolumn{4}{|l|}{ Malarial IPTp } \\
\hline Yes & 914 (93.4) & $923(93.0)$ & \multirow[t]{2}{*}{0.78} \\
\hline No & $65(6.6)$ & $69(7.0)$ & \\
\hline \multicolumn{4}{|l|}{ Syphilis } \\
\hline Negative & $830(84.8)$ & $869(87.6)$ & \multirow[t]{3}{*}{0.14} \\
\hline Positive & $11(1.1)$ & $6(0.6)$ & \\
\hline Missing & $138(14.1)$ & $117(11.8)$ & \\
\hline \multicolumn{4}{|l|}{ HIV status } \\
\hline Negative & $887(90.6)$ & 892 (89.9) & 0.55 \\
\hline Positive & $46(4.7)$ & $57(5.6)$ & \\
\hline Missing & $46(4.7)$ & $43(4.3)$ & \\
\hline Gestational age a & & & \\
\hline Median (IQR) & $39(38-40)$ & $39(38-40)$ & 0.80 \\
\hline Post-term gestatic & & & \\
\hline Yes & $41(4.2)$ & $54(5.4)$ & 0.19 \\
\hline No & $938(95.8)$ & $938(94.6)$ & \\
\hline Newborn weight & & & \\
\hline Median (IQR) & $3300(3000-3500)$ & $3300(3000-3500)$ & 0.70 \\
\hline
\end{tabular}

All analyses were conducted using Stata/SE (StataCorp, 2011. Stata Statistical Software: Release V.12. College Station, Texas, USA: StataCorp LP).
Role of funding source

The sponsor had no role in designing the study, analysing data, collecting data, interpreting the results, writing 
the report or in the decision to submit the paper for publication. The corresponding author had complete access to all the data.

\section{RESULTS}

From July 2012 to December 2013, we screened 2042 women antenatally. Fifty-three women were ineligible (50 planned to deliver elsewhere, 3 planned caesarean delivery); 2 women declined to participate; 1987 were enrolled (figure 1). After assignment to a monitoring method, 8 of the 987 in the Pinard arm were excluded from analysis (1 was lost to follow-up, 1 delivered before the partograph was started, 2 had undiagnosed breech births, 4 had undiagnosed multiple births); and 8 of the 1000 in the Doppler arm were excluded (3 delivered before the partograph was started, 3 had undiagnosed breech births, 2 had undiagnosed multiple births). The final study group was $\mathrm{n}=979$ in the Pinard arm and $\mathrm{n}=992$ in the Doppler arm.

Of the 1971 women analysed, the median maternal age was 26 years (IQR 24-30; table 1). There was a slightly higher though not statistically significant number of postterm women ( $\geq 42$ weeks of gestational age) in the Doppler versus the Pinard arm (54/992 (5.4\%) vs 41/979 (4.2\%), $\mathrm{p}=0.193)$. A similar proportion of women in the Doppler versus the Pinard arm were primiparous (395/992 (39.8\%) vs 413/979 (42.2\%)), with similar median gestational age (39 weeks, IQR 38-40), and similar median newborn weight (3300 g, IQR 3000-3500 g).

There were no differences between the study arms in Apgar score $<7$ at 5 min (23 (2.3\%) in the Doppler vs 17 $(1.7 \%)$ in the Pinard, $\mathrm{p}=0.40$ ) or admission to neonatal intensive care unit for any reason $(48(4.8 \%)$ in the Doppler vs $36(3.7 \%)$ in the Pinard, $\mathrm{p}=0.20)$. Similar proportions of women in the Doppler versus Pinard arm had caesarean deliveries $(175 / 992(17.6 \%)$ vs $166 / 979$ $(17.0 \%), \mathrm{p}=0.695)$.

There were significantly higher numbers of FHR abnormalities detected in the Doppler versus Pinard $\operatorname{arm}(75 / 992(7.6 \%)$ vs $46 / 979 \quad(4.7 \%), \quad \mathrm{p}=0.008$, IRR $=1.61,95 \%$ CI 1.13 to 2.30 ; table 2 ). There were higher though not statistically significant numbers of intrapartum stillbirths in the Doppler versus Pinard arm $(4 / 988(0.4 \%)$ vs $1 / 977(0.1 \%), p=0.184, I R R=3.94,95 \%$ CI 0.44 to 35.24), and higher numbers of neonatal deaths prior to discharge $(7 / 985(0.7 \%)$ vs $5 / / 973$ $(0.5 \%), \mathrm{p}=0.579, \mathrm{IRR}=1.38,95 \%$ CI 0.44 to 4.34$)$.

There were 121 cases of abnormal FHR detected in labour (figure 2). Of the 17 deaths in total (intrapartum stillbirths and neonatal deaths prior to discharge), 5 were associated with the detection of abnormal FHR in labour. In a subgroup analysis of those cases where abnormal FHR was detected, there was a higher though not statistically significant proportion of deaths in the Doppler versus Pinard arm (4/71 (5.3\%) vs $1 / 45$ (2.2\%), IRR $=2.45,95 \%$ CI 0.28 to 21.47$)$. Of the remaining 12 deaths that had a normal FHR reported, 3 had missing cause of death, 1 had a congenital anomaly and cause of death for the remaining 8 was intrauterine hypoxia, respiratory distress or NE, suggesting that an abnormal FHR was a missed diagnosis in labour for these 8 deaths.

\section{DISCUSSION}

Detection of abnormal FHR in labour is essential for identifying the fetus in need of responsive management such as prompt delivery. We report that intermittent auscultation with a Doppler identifies 60\% more cases in need of prompt delivery (IRR=1.61); however, we did not find that this identification resulted in a significant decrease in mortality, although one would expect that higher detection should lead to prompt delivery and improved outcomes.

We propose a number of explanations for this lack of detected impact. We considered that there may have been a learning curve for staff using the Doppler as a new technology; however, we found no difference in outcomes over time or between groups (data not shown).

\begin{tabular}{|c|c|c|c|c|c|}
\hline & Pinard $(n=979)$ & Doppler $(n=992)$ & p Value & IRR* $^{*}(95 \% \mathrm{Cl})$ & p Value \\
\hline \multicolumn{6}{|c|}{ Abnormality detected } \\
\hline Yes & $46(4.7)$ & $75(7.6)$ & 0.008 & 1.61 (1.13 to 2.30$)$ & 0.009 \\
\hline No & $933(95.3)$ & $917(92.4)$ & & & \\
\hline \multicolumn{6}{|c|}{ Intrapartum stillbirth } \\
\hline Yes & $1(0.1)$ & $4(0.4)$ & 0.184 & $3.94 \dagger(0.44$ to 35.24$)$ & 0.219 \\
\hline No & 977 (99.9) & 988 (99.6) & & & \\
\hline Missing & 1 & 0 & & & \\
\hline \multicolumn{6}{|c|}{ Neonatal death prior to discharge } \\
\hline Yes & $5(0.5)$ & $7(0.7)$ & 0.579 & $1.38 \dagger(0.44$ to 4.34$)$ & 0.552 \\
\hline No & $973(99.5)$ & $985(99.3)$ & & & \\
\hline Missing & 1 & & & & \\
\hline
\end{tabular}




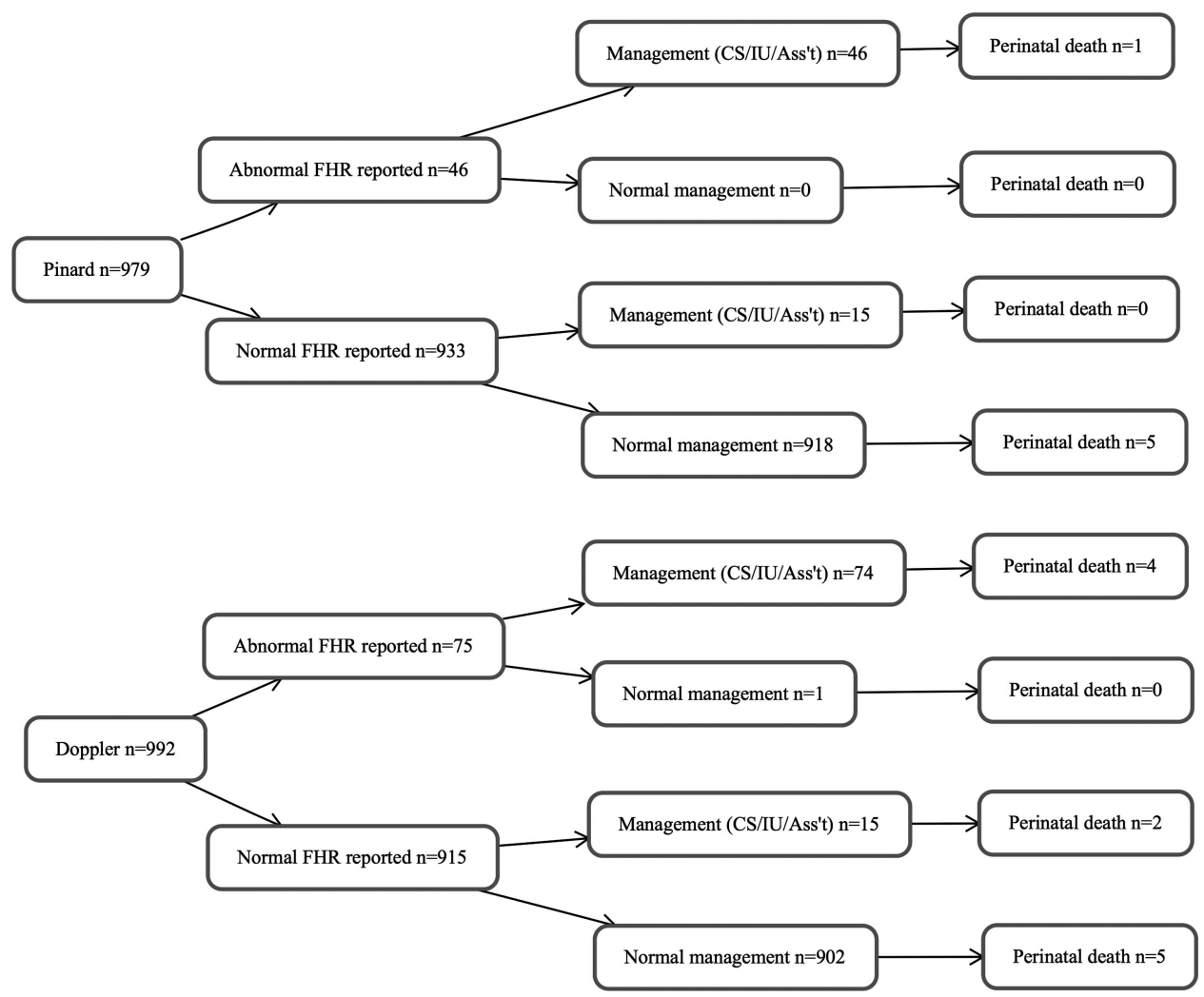

Figure 2 Flow diagram for outcome by fetal heart rate and management. FHR, fetal heart rate; CS/IU/Ass't, caesarean delivery, intrauterine resuscitation, assisted delivery.

Second, it is possible that use of technology such as the Doppler could lead to false assurance that FHR was being closely monitored, delayed involvement of senior staff and subsequent delivery, or there may simply have been delay between recognition and action, which, by chance, had more deleterious effects in the intervention group. Third, this study sample size and power were based on the Mahomed et al ${ }^{14}$ study, aiming to detect a $30 \%$ reduction in intrapartum stillbirth in the Doppler compared with the Pinard group and this may be optimistic, necessitating a larger sample size to demonstrate any improved outcomes, given the improved detection rates in the Doppler group.

Some study limitations include the fact that we were unable to perform secondary screening of suspected fetal hypoxia through the use of CTG and we could not confirm the presence of fetal hypoxia acidaemia via fetal blood scalp sampling and cord blood gases; therefore, we were unable to assess if the identification (or lack of identification) of abnormal FHR was correlated with the presence of fetal hypoxia acidaemia. In addition, we were unable to exclude some cases where the underlying cause of death was other than fetal hypoxia (eg, congenital anomalies, early onset sepsis) due to diagnostic limitations in differentially diagnosing these cases. Finally, the screening process was linked to the partograph, which has well-recognised limitations. ${ }^{18}$
In conclusion, routine monitoring with a handheld Doppler increases the proportion of fetuses identified in need of prompt delivery via the identification of FHR abnormalities in labour; however, we did not find evidence that this leads to a decrease in the incidence of intrapartum stillbirth or neonatal death. While assessing user satisfaction was not the objective of this study, the care providers and the women expressed preference for the Doppler, and given that the Doppler performed no worse than the Pinard in detecting abnormal FHR or in newborn survival, this should be an area of further research. Finally, this study demonstrates the need for a larger study with linkage to rapid response for abnormal FHR, including caesarean section to ensure that increased detection using the Doppler leads to decreased death and disability.

\section{Author affiliations}

${ }^{1}$ Department of Obstetrics and Gynaecology, St. Raphael of St. Francis Hospital Nsambya, Kampala, Uganda

${ }^{2}$ Centre for Global Child Health, Hospital for Sick Children, Toronto, Canada

${ }^{3}$ Powerfree Education and Technology, Cape Town, South Africa

${ }^{4}$ Department of Obstetrics and Gynaecology, Uganda Martyrs Hospital

Rubaga, Kampala, Uganda

${ }^{5}$ London School of Hygiene \& Tropical Medicine, London, UK

Acknowledgements The authors would like to thank all the midwives and staff of Nsambya Hospital maternity care services for their enthusiastic participation and all the women who participated for their willingness to be part of the trial. We are grateful to Hanifah Senguendo, Patrick Aligyira of 
Saving Newborn Lives, Save the Children Uganda and the members of Ugandan Obstetric Society and other colleagues for valuable inputs to the training materials. The authors appreciate the insights and helpful comments of Kate Kerber and Dave Woods.

Contributors All authors designed the study. RB, DGB, AJ and PM carried out data collection. DGB and ALM performed analysis and wrote the first draft of the manuscript. All authors reviewed and provided feedback on the manuscript draft.

Funding Grand Challenges Canada provided funding for the trial (grant number CRS1 0018) and Laerdal Foundation for the training module 'Helping Babies Survive Labour' (grant number 40038).

Competing interests $\mathrm{AJ}$ is a paid employee of Powerfree, the not-for-profit designers of the handheld Doppler used in this study.

Ethics approval Sickkids Research Ethics Board, Nsambya Internal Review Board, Uganda National Council for Science and Technology.

Provenance and peer review Not commissioned; externally peer reviewed.

Data sharing statement No additional data are available.

Open Access This is an Open Access article distributed in accordance with the Creative Commons Attribution Non Commercial (CC BY-NC 4.0) license, which permits others to distribute, remix, adapt, build upon this work noncommercially, and license their derivative works on different terms, provided the original work is properly cited and the use is non-commercial. See: http:// creativecommons.org/licenses/by-nc/4.0/

\section{REFERENCES}

1. Lawn JE, Blencowe H, Oza S, et al. Every newborn: progress, priorities, and potential beyond survival. Lancet 2014;384:189-205.

2. Lee AC, Kozuki N, Blencowe $\mathrm{H}$, et al. Intrapartum-related neonatal encephalopathy incidence and impairment at regional and global levels for 2010 with trends from 1990. Pediatr Res 2013;74(Suppl 1):50-72.

3. Lawn JE, Blencowe H, Pattinson R, et al. Stillbirths: Where? When? Why? How to make the data count? Lancet 2011;377:1448-63.

4. Lawn JE, Kinney M, Lee AC, et al. Reducing intrapartum-related deaths and disability: can the health system deliver? Int J Gynaecol Obstet 2009;107(Suppl 1):S123-40, S140-2.

5. SOGC. Advances in labour and risk management. Ottawa: Society of Obstetricians and Gynaecologists of Canada, 2013.
6. NICE. The Use of electronic fetal monitoring. NHS Clinical Guideline C, 2003;23807:1-17.

7. Liston R, Sawchuck D, Young D; Society of Obstetrics and Gynaecologists of Canada; British Columbia Perinatal Health Program. Fetal health surveillance: antepartum and intrapartum consensus guideline. J Obstet Gynaecol Can 2007;29: S3-56.

8. Hofmeyr GJ, Haws RA, Bergström S, et al. Obstetric care in low-resource settings: what, who, and how to overcome challenges to scale up? Int J Gynaecol Obstet 2009;107(Suppl 1): S21-44, S44-5.

9. Alfirevic Z, Devane D, Gyte GM. Continuous cardiotocography (CTG) as a form of electronic fetal monitoring (EFM) for fetal assessment during labour. Cochrane Database Syst Rev 2013;5: CD006066.

10. Mangesi L, Hofmeyr GJ, Woods D. Assessing the preference of women for different methods of monitoring the fetal heart in labour. S Afr J Obstet Gynaecol 2009;15:58-9.

11. Bezuidenhout $\mathrm{H}$, Woods $\mathrm{D}$, Wyatt $\mathrm{J}$, et al. Does fetal heart rate count? Developing a low cost, alternative powered Doppler fetal heart monitor for use in low resource high mortality settings. 4th IET Seminar on Appropriate Healthcare Technologies for Developing Countries; 2006:155-9.

12. Woods D. Appropriate technology and education for improved intrapartum care in underresourced countries. S Afr J Obstet Gynaecol 2009;15:78-9.

13. Devane D, Lalor JG, Daly S, et al. Cardiotocography versus intermittent auscultation of fetal heart on admission to labour ward for assessment of fetal wellbeing. Cochrane Database Syst Rev 2012;2:CD005122.

14. Mahomed K, Nyoni R, Mulambo T, et al. Randomised controlled trial of intrapartum fetal heart rate monitoring. $B M J$ 1994;308:497-500.

15. Ellis M, Manandhar N, Manandhar DS, et al. Risk factors for neonatal encephalopathy in Kathmandu, Nepal, a developing country: unmatched case-control study. BMJ 2000;320:1229-36.

16. Amiel-Tison C. Update of the Amiel-Tison neurologic assessment for the term neonate or at 40 weeks corrected age. Pediatr Neurol 2002;27:196-212.

17. Barros AJ, Hirakata VN. Alternatives for logistic regression in cross-sectional studies: an empirical comparison of models that directly estimate the prevalence ratio. BMC Med Res Methodol 2003;3:21.

18. Lavender T, Hart A, Smyth RM. Effect of partogram use on outcomes for women in spontaneous labour at term. Cochrane Database Syst Rev 2013;7:CD005461. 